\title{
An in vitro evaluation of Candida tropicalis infectivity using human cell monolayers
}

Correspondence

Mariana Henriques

mcrh@deb.uminho.pt

Received 10 February 2011

Accepted 9 May 2011

\author{
Melyssa Negri, Cláudia Botelho, Sónia Silva, \\ Luís Miguel Reis Henriques Lopes, Mariana Henriques, \\ Joana Azeredo and Rosário Oliveira
}

Institute for Biotechnology and Bioengineering, Centre of Biological Engineering, Universidade do Minho, Campus de Gualtar, 4710-057 Braga, Portugal

\begin{abstract}
The aim of this study was to investigate the interaction of Candida tropicalis with three different human cell lines: TCC-SUP (epithelial cells from urinary bladder), HeLa (epithelial cells from cervical carcinoma) and Caco-2 (epithelial cells from colorectal adenocarcinoma). In particular we sought to assess the degree of cell damage and activity reduction induced by $C$. tropicalis adhesion and the role of secreted aspartyl proteinase (SAP) gene expression in this process. Two C. tropicalis strains were used: the reference strain ATCC 750 and a clinical isolate from urine (U69). The ability of C. tropicalis to adhere to a confluent layer of human cells was determined using an adaptation of the crystal violet staining method; cell damage and cell activity inhibition induced by the adhesion of $C$. tropicalis were assessed by measuring lactate dehydrogenase and tetrazolium salt (MTS) reduction, respectively. C. tropicalis SAP gene expression was determined by real-time PCR. Both $C$. tropicalis strains were able to adhere to the different human cells, although in a strain- and cell-line-dependent manner. Concerning the cellular response to C. tropicalis, the highest inhibition of cell activity was obtained for Caco-2, followed by TCC-SUP and HeLa cells. The highest percentage of cell damage (around 14\%) was observed for TCC-SUP cells in contact with the U69 isolate and for Caco-2 in contact with the reference strain. Real-time PCR analysis revealed a wide range of expression profiles of SAP genes for both C. tropicalis strains in contact with the different types of epithelial cells. SAPT3 was the gene expressed at the highest level for both $C$. tropicalis strains in contact with the three human epithelial cell lines. The results highlight that the response of human cells to $C$. tropicalis adhesion, as well as production of SAPs, is dependent on both the strain and the epithelial cell line.
\end{abstract}

\section{INTRODUCTION}

Adhesion to host surfaces, such as human epithelial cells, and secretion of hydrolytic enzymes are considered important factors for Candida tropicalis virulence (Negri et al., 2010b; Okawa et al., 2008; Silva et al., 2011; Zaugg et al., 2001). The capacity of C. tropicalis to adhere to and infect host cells becomes a serious problem when patients are in intensive care units, mainly because this yeast is associated with higher dissemination potential and mortality, particularly in oncological patients (Chakrabarti et al., 2009; Kothavade et al., 2010; Nucci \& Colombo, 2007). In order to clarify the infectivity of Candida species, several in vitro studies have been performed using human cell monolayers from urinary, vaginal and intestinal epithelia (Bendel \& Hostetter, 1993; Negri et al., 2010b; Sohn et al., 2006).

In addition, hydrolytic enzymes such as secreted aspartyl proteinases (Saps) are known to facilitate invasion and

Abbreviations: CV, crystal violet; LDH, lactate dehydrogenase; MTS, 3-(4,5-dimethylthiazol-2-yl)-5-(3-carboxymethoxyphenyl)-2-(4-sulfophenyl) $-2 \mathrm{H}$-tetrazolium. colonization of tissues by disrupting the host mucosal membranes and by degrading important immunological and structural defence proteins (Rüchel et al., 1992; Zaugg et al., 2001). C. tropicalis possesses at least four genes encoding Saps; these are designated SAPT1, SAPT2, SAPT3 and SAPT4 (Zaugg et al., 2001).

Since $C$. tropicalis colonizes specific body sites such as the gastrointestinal and urinary tract and is associated with severe and invasive candidosis (Pfaller, 1992), it is important to understand the mechanisms of interaction between these epithelia and C. tropicalis. The aim of this work was to study the effect of $C$. tropicalis colonization of different human epithelial cells and the levels of expression of SAP genes by C. tropicalis when interacting with different human cells.

\section{METHODS}

Yeasts and growth conditions. Two strains of $C$. tropicalis were used in this study, a reference strain from the American Type Culture Collection (ATCC 750) and an isolate (U69) obtained from a urine 
sample of an 84-year-old female patient with candiduria admitted to the intensive care of an oncological unit and belonging to the archive collection of the University Hospital in Maringá, Paraná, Brazil. For each experiment, strains were inoculated in Sabouraud dextrose broth (SDB; Merck) and incubated for $18 \mathrm{~h}$ at $37^{\circ} \mathrm{C}$ with agitation at 120 r.p.m. After incubation, cells were harvested by centrifugation at $8000 \mathrm{~g}$ for $5 \mathrm{~min}$ at $4{ }^{\circ} \mathrm{C}$ and washed twice with phosphate buffered saline (PBS: $\mathrm{NaCl} 8 \mathrm{~g} \mathrm{l}^{-1}, \mathrm{KCl} 0.2 \mathrm{gl}^{-1}, \mathrm{Na}_{2} \mathrm{HPO}_{4} 1.44 \mathrm{gl}^{-1}, \mathrm{KH}_{2} \mathrm{PO}_{4}$ $\left.0.2 \mathrm{~g} \mathrm{l}^{-1} ; \mathrm{pH} 7.5\right)$.

Human epithelial cell lines. To assess the adhesion ability of C. tropicalis to human epithelial cells, the following cell lines were used as models: (i) TCC-SUP cells, derived from human urinary bladder epithelial cells (DSMZ - German Collection of Microorganisms and Cell Cultures); (ii) HeLa cells, derived from a human cervical carcinoma, donated by 'Instituto Gulbenkian de Ciência', Lisbon, Portugal; (iii) Caco- 2 cells, derived from a human colorectal adenocarcinoma cell line (ATCC HTB-37), kindly donated by Carla Nunes, Department of Biochemistry, Faculdade de Farmácia da Universidade de Coimbra, Coimbra, Portugal. Cells were cultured at $37{ }^{\circ} \mathrm{C}$ under $5 \% \mathrm{CO}_{2}$ in Dulbecco's modified Eagle's medium (D-MEM; Gibco) containing $10 \%$ fetal bovine serum (Gibco) and $1 \%$ penicillin/streptomycin (P/S; Gibco). After achieving $80 \%$ confluence, cells were detached using $25 \%$ trypsin-EDTA (Gibco) solution; the cell concentration was adjusted to

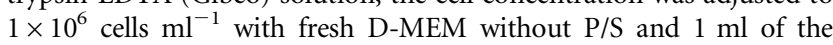
suspension was added to the wells of a 24 -well plate and incubated at $37{ }^{\circ} \mathrm{C}$ under $5 \% \mathrm{CO}_{2}$ for $24 \mathrm{~h}$. Prior to the adhesion assays, the wells were washed twice with PBS.

\section{Adhesion assay}

The yeast cells were suspended in D-MEM, without phenol red, to a final concentration of $1 \times 10^{7}$ yeast $\mathrm{ml}^{-1}$ using a Neubauer chamber (Boeco). Then, $1 \mathrm{ml}$ of this suspension was added to each well of a 24-well plate covered with a confluent layer of a human epithelial cell line. After $2 \mathrm{~h}$ incubation at $37{ }^{\circ} \mathrm{C}$ under $5 \% \mathrm{CO}_{2}$, each well was washed once with $\mathrm{PBS}$ to remove unattached yeasts.

C. tropicalis quantification. The adhered yeasts were quantified using the crystal violet $(\mathrm{CV})$ staining method, according to Negri et al. (2010a). The mean absorbance of yeasts was expressed as the absorbance per area of each well and standardized by the number of adhered yeasts per area of each well using a C. tropicalis standard curve (Negri et al., 2010a, b). All the procedures were repeated in triplicate in at least three separate assays.

Determination of percentage inhibition of cell activity. After the washing step with PBS, the remaining adhered yeasts were killed by incubating the well plates for $2 \mathrm{~h}$ and adding a $1 \%$ amphotericin $\mathrm{B}$ (AB) solution (Sigma, USA, $250 \mu \mathrm{g} \mathrm{ml}^{-1}$ ) in D-MEM without phenol red at $37{ }^{\circ} \mathrm{C}$ and $5 \% \mathrm{CO}_{2}$. The $\mathrm{AB}$ solution was then discarded and the epithelial cells' activity was determined using the CellTiter 96 assay (Promega), based on the reduction of MTS [3-(4,5-dimethylthiazol2-yl)-5-(3-carboxymethoxyphenyl)-2-(4-sulfophenyl)-2H-tetrazolium] with $1 \% \mathrm{AB}$ in D-MEM without phenol red. MTS is bioreduced by human epithelial cells into a formazan product that is soluble in tissueculture medium. After $2 \mathrm{~h}$ incubation at $37{ }^{\circ} \mathrm{C}$ in the dark, the absorbance of the formazan was measured at $490 \mathrm{~nm}$. A control was performed by measuring the cellular activity of human cells grown in the same conditions but in the absence of yeast cells. The effect of C. tropicalis on human epithelial cells was expressed as the percentage inhibition of cell activity standardized by the number of adhered yeast cells, with the MTS control corresponding to $100 \%$ activity of human cells, according to the following equation: percentage inhibition $=100 \times$ $\left.\left[\mathrm{MTS}_{\text {Control }}-\mathrm{MTS}_{\text {With C. tropicalis }}\right) / \mathrm{MTS}_{\text {Control }}\right] /$ No. of adhered yeasts.

All the procedures were repeated in triplicate in at least three separate assays.
Assay for epithelial cell damage. The release of lactate dehydrogenase $(\mathrm{LDH})$ by epithelial cells into the culture medium was used as a measure of cell damage. The LDH concentration in the medium was measured after $2 \mathrm{~h}$ of adhesion using the CytoTox-ONE kit (Promega), following the manufacturer's instructions. Two controls for LDH activity were prepared: (i) epithelial cells grown in the absence of $C$. tropicalis and (ii) yeast cells as sole culture. The LDH concentrations of both controls were subtracted from the LDH released by epithelial cells infected with yeasts. The effect of C. tropicalis on epithelial cells was expressed as the percentage of $\mathrm{LDH}$ released per number of adhered yeast cells, taking as $100 \%$ the concentration of LDH released by a completely killed epithelial cell monolayer (using the killing buffer provided with the kit), according to the following equation: percentage cell damage $=100 \times$ $\left\{\mathrm{LDH}_{\text {Assay }}-\left[\left(\mathrm{LDH}_{\text {Control (i) }}+\mathrm{LDH}_{\text {Control (ii) }}\right)\right] / \mathrm{LDH}_{\text {Killed epithelial cells }}\right\} /$ No. of adhered yeast cells.

All experiments were performed in triplicate.

\section{Analysis of SAP gene expression}

RNA extraction. Prior to RNA extraction, the adhered yeasts were removed with $500 \mu \mathrm{l}$ lysis buffer (Invitrogen) and transferred to screw-cap tubes (Bioplastics). Then glass beads $(0.5 \mathrm{~mm}$ diameter, approximately $500 \mu \mathrm{l}$ ) were added and the tubes were homogenized twice for $30 \mathrm{~s}$, using a Mini-BeadBeater-8 (Stratech Scientific). After disruption of the yeast cells, the PureLink RNA Mini kit (Invitrogen) was used for total RNA extraction according to the manufacturer's recommended protocol. To avoid potential DNA contamination the samples were treated with RNase-free DNase I (Invitrogen).

Primers. The primers used for real-time PCR (RT-PCR) were described by Silva et al. (2011) and their sequences are listed in Table 1.

Synthesis of cDNA. To synthesize the complementary DNA (cDNA) the iScript cDNA Synthesis kit (Bio-Rad) was used according to the manufacturer's instructions. For each sample $10 \mu \mathrm{l}$ extracted RNA was used.

Real-time PCR. Real-time PCR (CF X96 Real-Time PCR System; Bio$\mathrm{Rad}$ ) was used to determine the relative levels of SAPT1-4 mRNA transcripts, with actin $1(A C T 1)$ as a reference housekeeping gene. Each reaction mixture consisted of a working concentration of SsoFast EvaGreen Supermix (Bio-Rad), $300 \mathrm{nM}$ forward and reverse primer, and $1 \mu \mathrm{lDNA}$, in a final reaction volume of $20 \mu$ l. Negative controls (water) were included in each run. The relative quantification of SAPT1-4 gene expression was performed by the $\Delta C_{\mathrm{t}}$ method. Each reaction was performed in triplicate and mean values of relative expression were determined for each SAP gene.

Statistical analysis. The results obtained were analysed using the SPSS 18 (Statistical Package for the Social Sciences) program. Oneway ANOVA with the Bonferroni test was used to compare the number of yeasts adhering to epithelial cells, and cell activity and damage. All tests were performed with a confidence level of $95 \%$. All the experiments were performed in triplicate and in three independent assays.

\section{RESULTS}

The cellular activity and the degree of cell damage as a result of the interaction of $C$. tropicalis with the different human epithelial cell lines (TCC-SUP, HeLa and Caco-2) are presented in Table 2; the results for SAPT1-4 gene expression are presented in Table 3. In general, the 
Table 1. Primers used for real-time-PCR analysis of SAP and control $(A C T 1)$ gene expression

\begin{tabular}{|c|c|c|c|}
\hline Sequence $\left(5^{\prime} \rightarrow 3^{\prime}\right)$ & Primer & Target & PCR product size (bp) \\
\hline GGAAGATCTGATGTGCCAACTACATTGA & Forward & SAPT1 & 1005 \\
\hline CGTGCGGCCGCTCTACAAAGCCGAGATGTCT & Reverse & & \\
\hline TTCTTCTAGTGGTACCTGGGTCAAAG & Forward & SAPT2 & 762 \\
\hline CATAGATCTCTAAACAATAGTGACATTAGA & Reverse & & \\
\hline ACTTGGATTTCCAGCGAAGA & Forward & SAPT3 & 165 \\
\hline AGCCCTTCCAATGCCTAAAT & Reverse & & \\
\hline GTACTCGAGCTCCTACAACTTCACСТCСТ & Forward & SAPT4 & 1130 \\
\hline CATGGATCCCTATGTAAGTGGAAGTATGTT & Reverse & & \\
\hline GACCGAAGCTCCAATGAATC & Forward & ACT1 & 181 \\
\hline AATTGGGACAACGTGGGTAA & Reverse & & \\
\hline
\end{tabular}

C. tropicalis strains were able to adhere to the different epithelial cells and to cause a certain degree of cell damage and activity reduction. Moreover SAP genes were also expressed during epithelium colonization. However, these parameters were dependent on the yeast strain and on the epithelial cell line.

Curiously, strain U69, which is a clinical isolate from urine, adhered in significantly higher numbers $\left(2.45 \times 10^{6}\right.$ yeasts $\mathrm{cm}^{-2}$ ) to intestinal cells (Caco-2) than to urinary cells (TCC-SUP) (Fig. 1). In addition, this isolate induced a greater reduction in the cellular activity of intestinal cells than of urinary cells and showed the highest levels of SAPT1-3 gene expression (0.04, 0.03 and 6.52, respectively) when interacting with these cells. However, when in contact with urinary cells, strain U69 induced a greater percentage of cell damage $(14.24 \%)$ and a higher expression of SAPT4 (0.11) than when in contact with the other cell lines. The interaction of this urinary isolate with cervical cells (HeLa) resulted in low levels of both inhibition of cellular activity $(4.81 \%)$ and cell damage $(1.56 \%)$, and this strain expressed only SAPT3.

The reference strain (ATCC 750) adhered to TCC-SUP cells $\left(2.30 \times 10^{6}\right.$ yeasts $\left.\mathrm{cm}^{-2}\right)$, HeLa cells $\left(1.56 \times 10^{6}\right.$ yeasts $\left.\mathrm{cm}^{-2}\right)$ and Caco-2 cells $\left(1.48 \times 10^{6}\right.$ yeasts $\left.\mathrm{cm}^{-2}\right)$ to a similar extent $(P>0.05)$. Concerning the cellular response caused by this strain, the highest inhibition of cellular activity occurred in Caco-2 cells (50.27\%); the degree of cell damage (13.79\%) and expression of SAPT3 and 4 genes (9.71 and 0.49, respectively) were also highest for ATCC 750 when interacting with this line. HeLa cells showed less cellular response to the reference strain but higher expression of SAPT1 and 2 (0.06 and 0.05 , respectively).

It is important to highlight that both $C$. tropicalis strains affected HeLa cells to a lower extent than the other cell lines, causing low inhibition of cell activity and cell damage. SAPT3 was the gene that exhibited the highest level of expression.

\section{DISCUSSION}

C. tropicalis is an opportunistic human pathogen, which colonizes several anatomical sites, including skin and the gastrointestinal and genito-urinary tracts (Biasoli et al., 2002; Oksuz et al., 2007). Colonization by C. tropicalis, especially from specific body sites such as the gastrointestinal and urinary tracts, is associated with a high risk of development of infection (Pfaller, 1992). Several virulence factors seem to be responsible for C. tropicalis infections, which have high potential for dissemination, invasion and lethality (Krcmery \& Barnes, 2002; Okawa et al., 2008); these include the ability to adhere to human cells and to secrete enzymes such as proteases (GalánLadero et al., 2010; Negri et al., 2010b; Zaugg et al., 2001).

Table 2. Activity inhibition and damage of human cells evaluated by MTS and LDH, respectively, after C. tropicalis adhesion to three human cell lines

The $P$-value obtained from the comparison between the two strains is also presented. All values are means \pm SD.

\begin{tabular}{|c|c|c|c|c|c|c|}
\hline \multirow[t]{2}{*}{ Cell line } & \multicolumn{3}{|c|}{ Percentage inhibition of cell activity $( \pm S D)$} & \multicolumn{3}{|c|}{ Percentage cell damage $( \pm \mathrm{SD})$} \\
\hline & U69 isolate & Reference strain & $P$-value & U69 isolate & Reference strain & $P$-value \\
\hline TCC-SUP & $17.96( \pm 3.90)$ & $6.05( \pm 1.32)$ & $0.00^{*}$ & $14.24( \pm 3.73) \dagger$ & $11.07( \pm 2.17)$ & 0.35 \\
\hline $\mathrm{HeLa}$ & $4.81( \pm 0.91) \dagger$ & $5.12( \pm 0.99)$ & 1.00 & $1.56( \pm 0.54) \dagger$ & $2.39( \pm 0.59) \dagger$ & 1.00 \\
\hline Caco-2 & $31.53( \pm 3.91) \dagger$ & $50.27( \pm 2.60) \dagger$ & $0.00^{*}$ & $6.16( \pm 0.99)$ & $13.79( \pm 0.50) \dagger$ & $0.00^{*}$ \\
\hline
\end{tabular}

${ }^{\star}$ Statistically significant difference comparing between strains, but with the same cell lines $(P<0.05)$.

$\dagger$ Statistically significant difference comparing among cell lines, but with the same strain $(P<0.05)$. 
Table 3. Secreted aspartyl proteinase (SAPT1-4) gene expression associated with adhesion of C. tropicalis to three human cell lines

\begin{tabular}{|c|c|c|c|c|c|c|c|c|}
\hline \multirow[t]{2}{*}{ Cell line } & \multicolumn{8}{|c|}{ Relative expression of SAP genes* } \\
\hline & \multicolumn{4}{|c|}{ U69 isolate } & \multicolumn{4}{|c|}{ Reference strain } \\
\hline TCC-SUP & $0.02 \pm 0.01$ & $0.01 \pm 0.00$ & $5.01 \pm 0.63$ & $0.11 \pm 0.05$ & $0.04 \pm 0.00$ & $0.01 \pm 0.00$ & $0.77 \pm 0.85$ & $0.07 \pm 0.03$ \\
\hline HeLa & ND & ND & $0.09 \pm 0.00$ & ND & $0.06 \pm 0.04$ & $0.05 \pm 0.00$ & $5.91 \pm 0.37$ & $0.08 \pm 0.01$ \\
\hline
\end{tabular}

ND, No gene expression detected.

${ }^{\star}$ Mean $( \pm S D)$ arbitrary mRNA transcript levels in quantitative real-time PCR based upon triplicate measurements, presented as a percentage relative to the respective $A C T 1$ transcript level.

Several studies have been performed to clarify the behaviour of Candida species in the adhesion process, colonization and infection using human cell lines as a study model for Candida pathogenesis ex vivo (Bendel, 2003; Negri et al., 2010b; Pacheco et al., 2007; Saegusa et al., 2007; Sohn et al., 2006). However, compared to Candida albicans, only a few investigations have been performed to assess the virulence of $C$. tropicalis, particularly in the presence of different human cell lines. Thus, the major goal of the present study was to investigate, ex vivo, the potential of $C$. tropicalis to colonize and damage urinary, vaginal and intestinal epithelium. Therefore, three different cell lines were used: one from human bladder (TCC-SUP), one from human cervical carcinoma (HeLa), and one from human colorectal adenocarcinoma (Caco-2). These cell lines are very often used to study, in vitro, mechanisms of interaction between Candida and the gastrointestinal and genito-urinary tracts (Bendel \& Hostetter, 1993; Negri et al., 2010a, b; Sohn et al., 2006).

In the present study, and corroborating other authors, C. tropicalis yeasts were able to adhere to and to damage all three epithelial cell lines used (Bendel \& Hostetter, 1993;

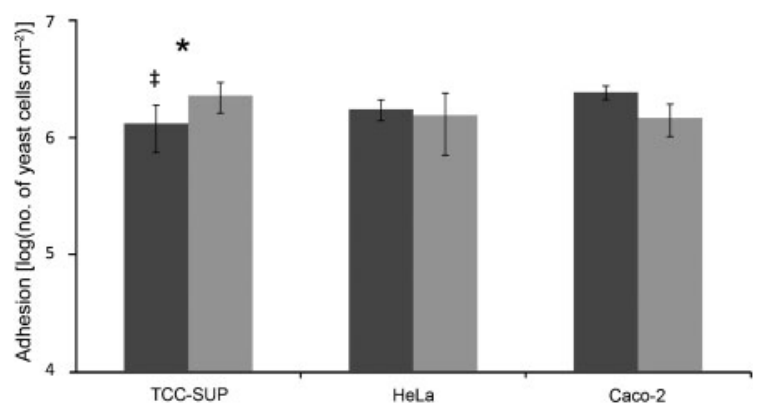

Fig. 1. C. tropicalis adhesion to the three cell lines (TCC-SUP, HeLa and Caco-2), analysed by the CV staining method. Dark grey bars, U69; light grey bars, reference strain (ATCC 750). *Isolates with statistically significant difference in extent of adhesion $(P<0.05)$. $\ddagger C$. tropicalis adhesion to cell line statistically different from other cell lines $(P<0.05)$. Error bars represent SD.
Negri et al., 2010a; Saegusa et al., 2007). Furthermore C. tropicalis showed a range of expression profiles of SAP genes (Silva et al., 2011; Zaugg et al., 2001), although in a strain- and cell-line-dependent manner (Negri et al., 2010b; Okawa et al., 2008). In previous studies, C. tropicalis strains have shown different behaviours in their ability to invade human tissue, with dramatic histopathological tissue alteration (Jayatilake et al., 2006; Okawa et al., 2008; Saegusa et al., 2007; Silva et al., 2011).

Comparing our results with other studies (Bendel, 2003; Negri et al., 2010b; Silva et al., 2011), shows that Candida species do not adhere to the same extent to the different types of mucosal cells, and also that there is not a strong correlation between the ability to adhere to a particular type of epithelial cells and the tissue site from which the yeast was isolated. It is important to highlight that the two strains used in this study, U69 and the reference strain, are from different clinical specimens and showed different infectivities. Strain U69 is a clinical isolate from a urine sample of a female patient from an oncology unit; the reference strain, ATCC 750, is a clinical isolate from a patient with bronchomycosis. Interestingly, strain U69 adhered to a higher extent to Caco-2 cells than to the other human cells and the reference strain adhered in similar extents to the different cell lines; curiously, it adhered in higher number to the urinary cell line TCC-SUP than did strain U69 (Fig. 1). Sohn et al. (2006) reported that the interaction of C. albicans with Caco-2 cells is distinct from the interaction with an epidermoid vulvo-vaginal cell line (A-431) and these authors suggested that this fact can be related to epithelial cells' morphology and molecular events occurring during adhesion. Furthermore, in a previous study with the same strains (Negri et al., 2010b), it was confirmed that $C$. tropicalis isolate U69 adhered to the TCCSUP cell line in lower numbers than the reference strain.

The cellular response to $C$. tropicalis adhesion was also studied, and interestingly both cell activity and integrity were affected. Although there is no direct correlation between inhibition of cell activity and cell damage, it was noticed (Table 2) that when cell inhibition was significantly high, cell damage was also more severe. To the authors' 
knowledge previous studies have only evaluated cell damage (by LDH measurement) or cell activity inhibition but never both approaches. Pacheco et al. (2007) showed that Candida famata caused a low inhibition of cell activity after $2 \mathrm{~h}$ of contact, in contrast to C. tropicalis strains adhered to Caco-2 cells, which showed high inhibition of cell activity. Opportunistic pathogenic yeasts, such as $C$. tropicalis, are able to promote cellular immune response and progressive cell damage during infection (Okawa et al., 2008; Saegusa et al., 2007; Silva et al., 2011). Moreover, Silva et al. (2011) demonstrated that C. tropicalis strains were able to cause significant tissue damage, measured by LDH release, after long periods of yeast contact with cells (12-24 h). Unfortunately, there are few studies regarding the effect of Candida species on the activity of human cells. Therefore, the present study is a step forward for the understanding of $C$. tropicalis pathogenesis.

Among the three cell lines, the lowest damage was observed in HeLa cells, highlighting that cell damage is dependent on the cell line. According to Sohn et al. (2006) C. albicans adhesion to epidermoid vulvo-vaginal cells seemed to occur more slowly than adhesion to Caco-2 cells and hyphal penetration into monolayers of the cell lines started after $4 \mathrm{~h}$, leading to damage of the cellular substrate and marking the beginning of the tissue invasion phase. Injury of host cells by Candida has been described as a complex mechanism of interaction between yeasts and host cells (Filler \& Sheppard, 2006; Silva et al., 2011; Sohn et al., 2006). However, this is mainly related to the time of infection, morphogenesis of Candida, morphology of host cells, and production of hydrolytic enzymes.

C. tropicalis adhesion to the epithelial cells also affected SAP gene expression. During the process of adhesion and invasion of host tissues, Candida species are known to secrete hydrolytic enzymes that damage the host cells' membrane integrity, leading to dysfunction or disruption of host structures (Zaugg et al., 2001). Furthermore, the expression of SAP genes by $C$. tropicalis has also been demonstrated during penetration of tissues, and evading macrophages after phagocytosis of yeast cells (Monod et al., 2002; Togni et al., 1991; Zaugg et al., 2001).

In this study, Caco-2 cells showed the highest inhibition of activity when in contact with each of the tested strains (Table 2), and strain U69 and the reference strain expressed the highest levels of SAPT1-3 and SAPT3-4, respectively, when interacting with these cells (Table 3 ). However, strain U69 in contact with TCC-SUP cells promoted a greater percentage of cell damage and a higher expression of SAPT4 and the same happened with the reference strain when in contact with Caco-2 cells. Studies with C. albicans indicate that each $S A P$ gene may be related to the yeast form and to a specific function (Monod et al., 2002; Naglik et al., 2003; Silva et al., 2011; Yang, 2003; Zaugg et al., 2001). Recent data for C. albicans indicated that SAP1SAP3 genes are expressed by yeast cells only and contribute to the adhesion to human cells and tissue damage, whereas
C. albicans SAP4 expression is confined to hyphae, and has been further implicated in systemic infection and in the evasion of phagocytosis (Yang, 2003).

Among the SAP genes, SAPT3 showed the highest level of expression for both strains (Table 3). Silva et al. (2011) studied the expression profiles of SAP genes for seven C. tropicalis strains in contact with reconstituted human oral epithelium and also determined the expression of the four genes, but SAPT2 and SAPT4 transcripts were detected to a similar extent to SAPT3. This difference in results may be related to the epithelium type and also to the different C. tropicalis strains studied. In the present case, the SAP gene expression was strain and human cell line dependent. It is important to highlight that only a few studies (Silva et al., 2011; Zaugg et al., 2001) have reported C. tropicalis $S A P T$ gene expression during adhesion to human cells and there is also limited knowledge about the role of these enzymes in $C$. tropicalis adhesion and tissue damage.

In summary, this study shows that $C$. tropicalis is able to adhere to different human cells, influencing their response in a manner dependent on cell type and yeast strain. Our results also stress the importance of using more than one methodology to assess cell injury caused by Candida species. Cell damage and activity caused by C. tropicalis seem to be related to the expression of different SAP genes. As a preliminary study, this work only explored a short period of contact between yeasts and epithelial cells; more studies, including longer contact times, would be of interest, as would the inclusion of different clinical isolates. Greater knowledge of the response of human cells to Candida stimuli could help in the development of new therapeutic strategies.

\section{ACKNOWLEDGEMENTS}

The authors acknowledge Coordenação de Aperfeiçoamento de Pessoal de Nível Superior (CAPES), Brazil, for supporting M.N.'s work through the grant BEX 4642/06-6 and FCT, Portugal, for supporting C. B. (SFRH/BPD/20987/2004) and S.S. (SFRH/BD/ 28341/2006). The authors would like to acknowledge Ana Rita Costa for the revision of the written English.

\section{REFERENCES}

Bendel, C. M. (2003). Colonization and epithelial adhesion in the pathogenesis of neonatal candidiasis. Semin Perinatol 27, 357-364.

Bendel, C. M. \& Hostetter, M. K. (1993). Distinct mechanisms of epithelial adhesion for Candida albicans and Candida tropicalis. Identification of the participating ligands and development of inhibitory peptides. J Clin Invest 92, 1840-1849.

Biasoli, M. S., Tosello, M. E. \& Magaró, H. M. (2002). Adherence of Candida strains isolated from the human gastrointestinal tract. Mycoses 45, 465-469.

Chakrabarti, A., Chatterjee, S. S., Rao, K. L. N., Zameer, M. M., Shivaprakash, M. R., Singhi, S., Singh, R. \& Varma, S. C. (2009). Recent experience with fungaemia: change in species distribution and azole resistance. Scand J Infect Dis 41, 275-284. 
Filler, S. G. \& Sheppard, D. C. (2006). Fungal invasion of normally non-phagocytic host cells. PLoS Pathog 2, e129.

Galán-Ladero, M. A., Blanco, M. T., Sacristán, B., FernándezCalderón, M. C., Pérez-Giraldo, C. \& Gómez-Garcia, A. C. (2010). Enzymatic activities of Candida tropicalis isolated from hospitalized patients. Med Mycol 48, 207-210.

Jayatilake, J. A., Samaranayake, Y. H., Cheung, L. K. \& Samaranayake, L. P. (2006). Quantitative evaluation of tissue invasion by wild type, hyphal and SAP mutants of Candida albicans, and non-albicans Candida species in reconstituted human oral epithelium. J Oral Pathol Med 35, 484-491.

Kothavade, R. J., Kura, M. M., Valand, A. G. \& Panthaki, M. H. (2010). Candida tropicalis: its prevalence, pathogenicity and increasing resistance to fluconazole. J Med Microbiol 59, 873-880.

Krcmery, V. \& Barnes, A. J. (2002). Non-albicans Candida spp. causing fungaemia: pathogenicity and antifungal resistance. $J$ Hosp Infect 50, 243-260.

Monod, M., Capoccia, S., Léchenne, B., Zaugg, C., Holdom, M. \& Jousson, O. (2002). Secreted proteases from pathogenic fungi. Int $J$ Med Microbiol 292, 405-419.

Naglik, J. R., Challacombe, S. J. \& Hube, B. (2003). Candida albicans secreted aspartyl proteinases in virulence and pathogenesis. Microbiol Mol Biol Rev 67, 400-428.

Negri, M., Gonçalves, V., Silva, S., Henriques, M., Azeredo, J. \& Oliveira, R. (2010a). Crystal violet staining to quantify Candida adhesion to epithelial cells. Br J Microbiol 67, 120-125.

Negri, M., Martins, M., Henriques, M., Svidzinski, T. I., Azeredo, J. \& Oliveira, R. (2010b). Examination of potential virulence factors of Candida tropicalis clinical isolates from hospitalized patients. Mycopathologia 169, 175-182.

Nucci, M. \& Colombo, A. L. (2007). Candidemia due to Candida tropicalis: clinical, epidemiologic, and microbiologic characteristics of 188 episodes occurring in tertiary care hospitals. Diagn Microbiol Infect Dis 58, 77-82.
Okawa, Y., Miyauchi, M. \& Kobayashi, H. (2008). Comparison of pathogenicity of various Candida tropicalis strains. Biol Pharm Bull 31, 1507-1510.

Oksuz, S., Sahin, I., Yildirim, M., Gulcan, A., Yavuz, T., Kaya, D. \& Koc, A. N. (2007). Phospholipase and proteinase activities in different Candida species isolated from anatomically distinct sites of healthy adults. Jpn J Infect Dis 60, 280-283.

Pacheco, M., Pisa, D., García-Gómez, P., Carrasco, L. \& Juarranz, Á. (2007). Attachment and entry of Candida famata in monocytes and epithelial cells. Microsc Res Tech 70, 975-986.

Pfaller, M. A. (1992). Laboratory aids in the diagnosis of invasive candidiasis. Mycopathologia 120, 65-72.

Rüchel, R., de Bernardis, F., Ray, T. L., Sullivan, P. A. \& Cole, G. T. (1992). Candida acid proteinases. J Med Vet Mycol 30 (Suppl. 1), 123-132.

Saegusa, S., Totsuka, M., Kaminogawa, S. \& Hosoi, T. (2007). Cytokine responses of intestinal epithelial-like Caco-2 cells to nonpathogenic and opportunistic pathogenic yeasts in the presence of butyric acid. Biosci Biotechnol Biochem 71, 2428-2434.

Silva, S., Hooper, S. J., Henriques, M., Oliveira, R., Azeredo, J. \& Williams, D. W. (2011). The role of secreted aspartyl proteinases in Candida tropicalis invasion and damage of oral mucosa. Clin Microbiol Infect 17, 264-272.

Sohn, K., Senyürek, I., Fertey, J., Königsdorfer, A., Joffroy, C., Hauser, N., Zelt, G., Brunner, H. \& Rupp, S. (2006). An in vitro assay to study the transcriptional response during adherence of Candida albicans to different human epithelia. FEMS Yeast Res 6, 1085-1093.

Togni, G., Sanglard, D., Falchetto, R. \& Monod, M. (1991). Isolation and nucleotide sequence of the extracellular acid protease gene $(A C P)$ from the yeast Candida tropicalis. FEBS Lett 286, 181-185.

Yang, Y. L. (2003). Virulence factors of Candida species. J Microbiol Immunol Infect 36, 223-228.

Zaugg, C., Borg-Von Zepelin, M., Reichard, U., Sanglard, D. \& Monod, M. (2001). Secreted aspartic proteinase family of Candida tropicalis. Infect Immun 69, 405-412. 